\title{
Crowd behavior classification based on generic descriptors
}

\begin{abstract}
Crowd behavior analysis plays an important role in high security interests in public areas such as railway stations, shopping centres, and airports, where large populations gather. The crowded scenes vary in various densities, structures and occlusion. It brings enormous challenges in identifying generic descriptors to describe motion dynamics caused by pedestrians walk in different directions with extremely diverse behaviors. Therefore, this research is proposal an approach for crowd behavior analysis to recognize the common properties across different crowded scenes. The recognized common properties are then used to identify generic descriptors from group-level for crowd behavior classification.
\end{abstract}

Keyword: Crowd behavior classification; Generic descriptors; Crowded scenes; Group-level; Motion 